\title{
Dynamic active earth pressure on retaining structures
}

\author{
DEEPANKAR CHOUDHURY and SANTIRAM CHATTERJEE \\ Department of Civil Engineering, Indian Institute of Technology - Bombay, \\ Powai, Mumbai 400 076, India \\ e-mail: dc@civil.iitb.ac.in; santiram@iitb.ac.in
}

MS received 3 January 2006; revised 23 June 2006

\begin{abstract}
Earth-retaining structures constitute an important topic of research in civil engineering, more so under earthquake conditions. For the analysis and design of retaining walls in earthquake-prone zones, accurate estimation of dynamic earth pressures is very important. Conventional methods either use pseudo-static approaches of analysis even for dynamic cases or a simple single-degree of freedom model for the retaining wall-soil system. In this paper, a simplified two-degree of freedom mass-spring-dashpot (2-DOF) dynamic model has been proposed to estimate the active earth pressure at the back of the retaining walls for translation modes of wall movement under seismic conditions. The horizontal zone of influence on dynamic earth force on the wall is estimated. Results in terms of displacement, velocity and acceleration-time history are presented for some typical cases, which show the final movement of the wall in terms of wall height, which is required for the design. The non-dimensional design chart proposed in the present study can be used to compute the total dynamic earth force on the wall under different input ground motion and backfill conditions. Finally, the results obtained have been compared with those of the available Scott model and the merits of the present results have been discussed.
\end{abstract}

Keywords. Earth pressure; 2-DOF dynamic model; translation; wall movement; earthquake.

\section{Introduction}

For the safe and economic design of retaining structures, correct estimation of earth pressure on retaining structures, sheet pile walls, braced excavation etc. is very important to civil engineers, especially geotechnical engineers. Due to its complexity in analysis, this problem has drawn the attention of researchers through the decades. Even under static conditions this is one of the most critical and complex problems of soil mechanics and geotechnical engineering. So, under dynamic condition and/or under seismic loading, the problem is no doubt challenging. The recent devastating earthquakes in India, like the Kashmir Earthquake in 2005, and the Bhuj Earthquake in 2001 have added important dimensions to this problem, as in the hilly

A list of symbols is given at the end of the paper 
regions, retaining structures are of utmost importance. Among the theories available till date for the estimation of seismic earth pressure, the Mononobe-Okabe $(1926,1929)$ method, which is the pioneering work in this field, is commonly used. This theory is based on a pseudostatic forced-based approach and hence is only dependent on the maximum amplitude, not on the frequency of ground motion. It also assumes relative movements of the wall and soil, large enough to induce a limit or failure state in the soil, and hence full mobilization of earth pressure is assumed in the analysis. Recently, researchers like Saran \& Gupta (2003); Choudhury \& Singh (2006) also proposed the pseudo-static force-based methods to estimate the seismic-active earth pressure behind a retaining wall. However, in practical cases, this movement may not always, achieve the limit state even for the static case. Recent work by Choudhury \& Subba Rao (2002) and Subba Rao et al (2004) has shown that even under static condition, the partial mobilization of earth pressure is more common than the full mobilization of earth pressure depending on the displacement of the wall. For small translational modes of wall movement, Scott (1973) first proposed a simplified mathematical model for estimating the response of both semi-infinite system and system bounded by two boundaries for the seismic-active state of earth pressure. In this model, the far-field response is estimated from the shearing resistance of a vertical shear-beam excited in similar fashion. Ground motion is assumed to be resisted by the shearing action of the vertical shear beam and by extensional, column-like behaviour between the far-field and the wall. The shearing resistance of the soil, close to the wall, is presumed to contribute nothing in this case, which as shown by researchers like Veletsos \& Younan (1994a) may have important effects on the resulting earth pressure. Another shortcoming of Scott's model is its total dependency on the stiffness of the springs between the far-field and the wall. As it assumes that the resulting active earth pressure is directly proportional to the stiffness of the set of the assumed springs, any error in estimating stiffness would result in an erroneous value of active earth pressure. Wood (1973) also gave an analytically correct solution of this problem through the finite element technique for soil retained between two vertical walls by rectifying the error in Scott's (1973) model.

Though several researchers have later modified Scott's (1973) model, most of them are too complicated to use for engineering practices. Also, the previous mathematical model of many researchers like Veletsos \& Younan (1994b) deals with the simplest and crude singledegree freedom model to estimate the seismic earth pressure on the retaining wall. Hence, the shortcomings of the mathematical model in estimating the seismic active earth pressure can be observed from the available literature.

In the present analysis, a simplified two-degree freedom mass-spring-dashpot (2-DOF) dynamic model for both wall and soil has been proposed as representing a semi-infinite soil medium retained by a vertical wall along one of its vertical boundaries and bonded to a rigid base. Response due to the input harmonic ground motion is then obtained and nondimensional results in graphical form are presented to compute the total seismic-active earth pressure under different damping properties of the soil and frequency ratio. The present results are compared with those of Scott's (1973) model.

\section{Soil-wall system and proposed mathematical model}

\subsection{Soil-wall system considered}

The soil-wall system considered is shown in figure 1. The semi-infinite, homogeneous, viscoelastic medium of soil is retained by a vertical rigid retaining wall along one of its vertical 




Figure 1. Soil-wall system considered.

boundaries, connected to a rigid base. The upper surfaces of the soil layer and the wall are assumed to be free. The base of the soil layer is excited by a harmonic excitation to simulate earthquake conditions. The material of the wall and the soil layer is defined by the mass density $\rho$, shear modulus of elasticity $G$, Poisson's ratio $\mu$, and the material damping factor $\eta$ of concrete and dense sand respectively. The material damping factor is assumed to be independent of the input frequency.

\subsection{Proposed mathematical model}

The system above is modelled by a simple two-degree freedom (2-DOF) mass-spring-dashpot dynamic model, as shown in figure 2 . The soil layer, i.e. mass $M_{1}$, is excited with a ground harmonic acceleration with amplitude $\ddot{x}_{g}$. The free-body diagrams of both the soil and wall masses are shown in figures 3 and 4 respectively. Considering the dynamic equilibrium of these two masses by using D'Alembert's principle, basic dynamic equations in canonical form can be obtained.

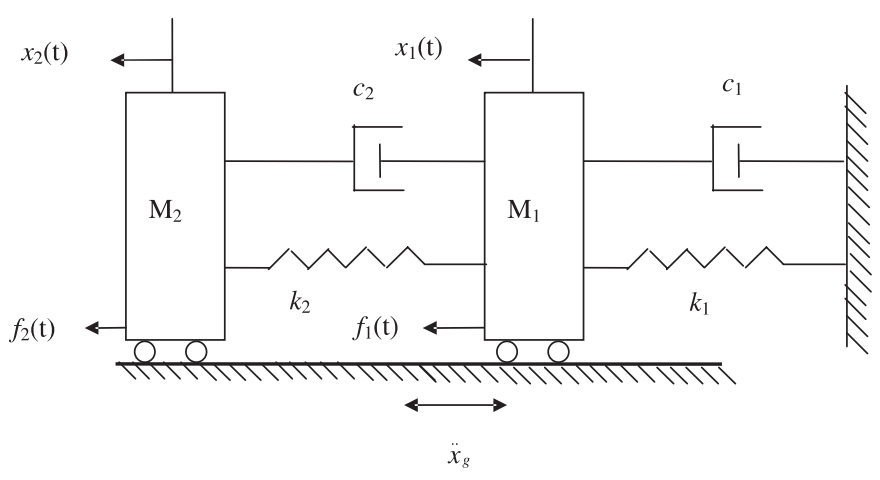

Figure 2. Proposed 2-DOF mathematical model. 


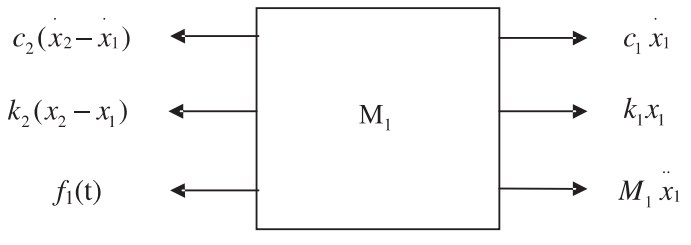

Figure 3. Free-body diagram of soil mass $M_{1}$.

\subsection{Free-body diagram of soil mass}

Considering dynamic equilibrium and using D'Alembert's principle, from figure 3 we can write,

$$
M_{1} \ddot{x}_{1}+c_{1} \dot{x}_{1}+c_{2}\left(\dot{x}_{1}-\dot{x}_{2}\right)+k_{1} x_{1}+k_{2}\left(x_{1}-x_{2}\right)=f_{1}(t)
$$

where, $x_{1}$ and $x_{2}$ are the displacements of masses $M_{1}$ and $M_{2}$ respectively; $\ddot{x}_{1}$ and $\ddot{x}_{2}$ are the velocities of masses $M_{1}$ and $M_{2}$ respectively; $\ddot{x}_{1}$ is the acceleration of mass $M_{1}$ and $f_{1}(t)$ is the applied external force on mass $M_{1}$, which is equal to $\left(M_{1} k_{h} g \sin \omega t\right)$ up to a finite time, $t_{1}$, which represents the duration of an earthquake and $f_{1}(t)$ is equal to zero after $t_{1}$.

\subsection{Free body diagram of wall mass}

Considering dynamic equilibrium and using D'Alembert's principle, from figure 4 we can write,

$$
M_{2} \ddot{x}_{2}+c_{2}\left(\dot{x}_{2}-\dot{x}_{1}\right)+k_{2}\left(x_{2}-x_{1}\right)=f_{2}(t)=0,
$$

where, $\ddot{x}_{2}$ is the acceleration of mass $M_{2}$ and $f_{2}(t)$ is the applied external force on mass $M_{2}$ which is equal to zero.

Combining (1) and (2), the basic dynamic equation can be written in matrix form:

$$
\begin{aligned}
& \left(\begin{array}{cc}
M_{1} & 0 \\
0 & M_{2}
\end{array}\right)\left\{\begin{array}{l}
\ddot{x}_{1} \\
\ddot{x}_{2}
\end{array}\right\}+\left(\begin{array}{cc}
\left(c_{1}+c_{2}\right) & -c_{2} \\
-c_{2} & c_{2}
\end{array}\right)\left\{\begin{array}{l}
\dot{x}_{1} \\
\dot{x}_{2}
\end{array}\right\}+\left(\begin{array}{cc}
\left(k_{1}+k_{2}\right) & -k_{2} \\
-k_{2} & k_{2}
\end{array}\right)\left\{\begin{array}{l}
x_{1} \\
x_{2}
\end{array}\right\} \\
& =\left\{\begin{array}{c}
f_{1}(t) \\
0
\end{array}\right\}
\end{aligned}
$$

The solution of (3) gives the displacement, velocity and acceleration response for both soil and wall. It can easily be seen from figure 4 that the resulting earth force magnitude on the wall, $\left|Q_{b}\right|$ can be obtained by using (4),

$$
\left|Q_{b}\right|=M_{2} \ddot{x}_{2}+c_{2}\left(\dot{x}_{2}-\dot{x}_{1}\right)+k_{2}\left(x_{2}-x_{1}\right) .
$$

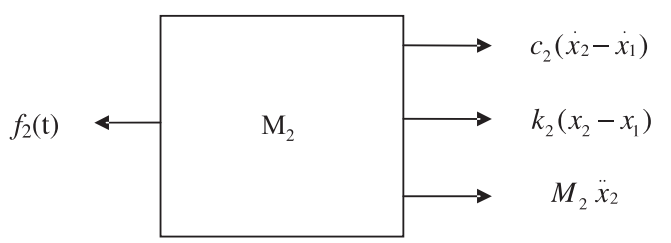

Figure 4. Free-body diagram of wall mass $M_{2}$. 


\section{Analysis of results}

For the solution of (3), Newmark's (see Chopra 2003) numerical technique for solving differential equations was adopted and a FORTRAN code was developed. To represent the extent of the semi-infinite soil stratum in the horizontal direction, an iterative procedure is used to obtain the critical finite minimum distance from the face of the wall such that the influence zone in the soil media is considered properly. It has been found by the iteration process that a distance of $10 H$, where $H$ is the vertical height of the wall, can be taken as the minimum horizontal distance of influence from the face of the wall. Further increase of this influence zone does not contribute much to the resulting earth force, $\left|Q_{b}\right|$ on the wall.

To obtain a solution, the concerned design parameters, like stiffness, $k$ and damping constant, $c$ are considered to be independent of the applied frequency of excitation. For the estimation of the stiffness value for both soil and wall, the method described by Veletsos \& Younan (1994b) was adopted. It is determined such that the undamped natural frequency of the model equals the fundamental natural frequency of the medium idealized as a series of vertical shear-beams. The stiffness, $k$ of a particular system can be estimated as,

$$
k=m\left(\pi^{2} / 4 H^{2}\right)(G / \rho),
$$

where $m$ is the mass of the system considered.

Solving the basic differential (3), the displacement, velocity and acceleration-time history response for any input dynamic motion is obtained. In figure 5, a typical result of
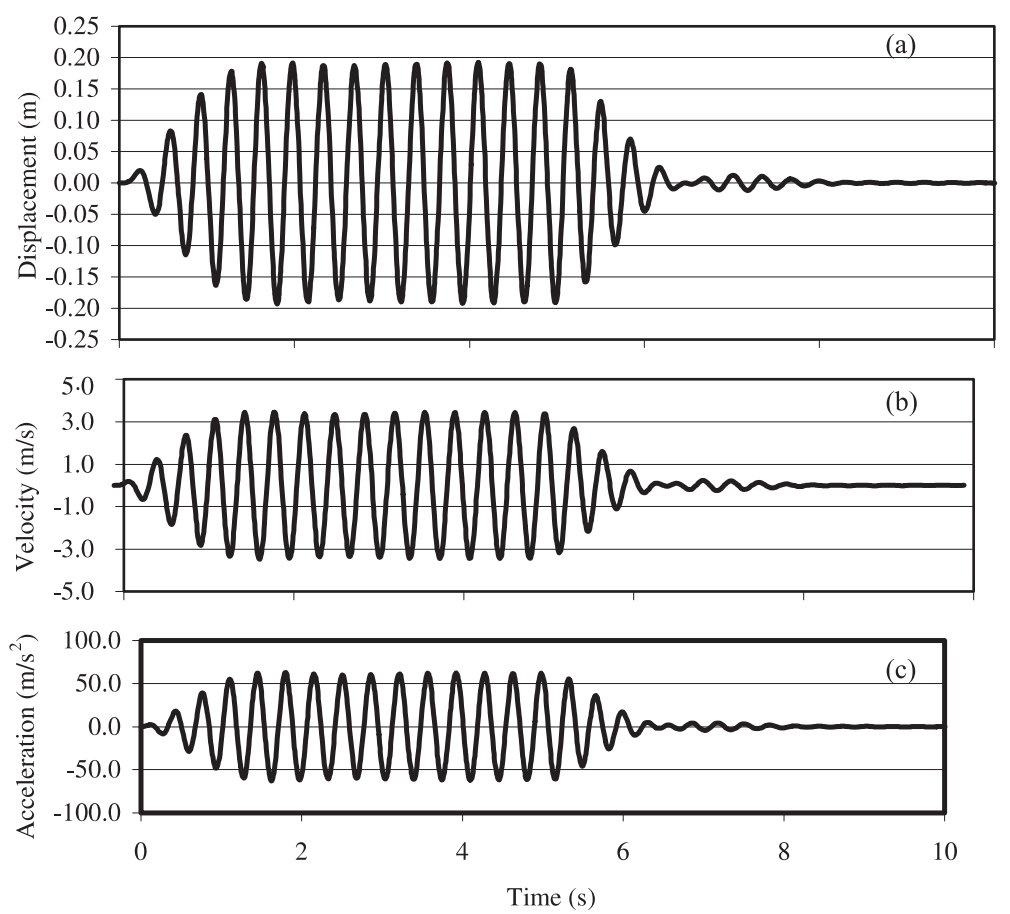

Figure 5. (a) Displacement, (b) velocity and (c) acceleration-time history response with, $H=5 \mathrm{~m}, \gamma_{\text {conc }}=24 \mathrm{kN} / \mathrm{m}^{3}, G_{\text {conc }}=9921 \times 10^{3} \mathrm{kN} / \mathrm{m}^{2}, \mu_{\text {conc }}=0.26, \eta_{\text {conc }}=5 \%, \gamma_{\text {soil }}=18 \mathrm{kN} / \mathrm{m}^{3}$, $G_{\text {soil }}=5769 \mathrm{kN} / \mathrm{m}^{2}, \mu_{\text {soil }}=0 \cdot 3, \eta_{\text {soil }}=20 \%$ and $k_{h}=0 \cdot 32$. 

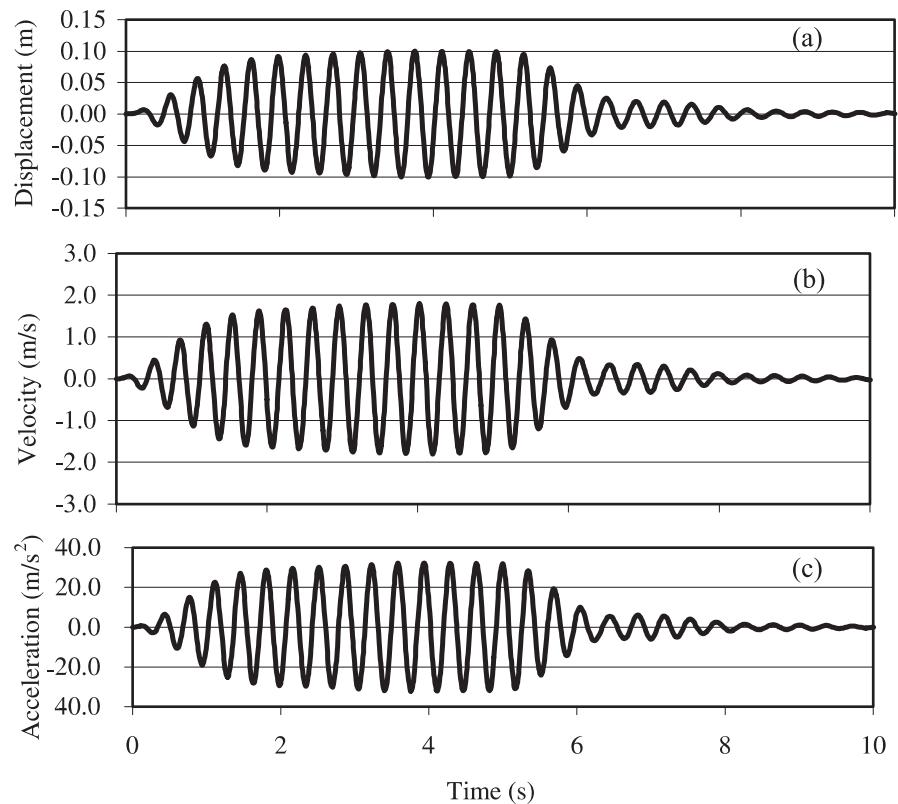

Figure 6. Displacement, velocity and acceleration-time history response with, $H=5 \mathrm{~m}, \gamma_{\text {conc }}=24 \mathrm{kN} / \mathrm{m}^{3}, G_{\text {conc }}=9921 \times 10^{3} \mathrm{kN} / \mathrm{m}^{2}, \mu_{\text {conc }}=0.26, \eta_{\text {conc }}=5 \%, \gamma_{\text {soil }}=18 \mathrm{kN} / \mathrm{m}^{3}$, $G_{\text {soil }}=5769 \mathrm{kN} / \mathrm{m}^{2}, \mu_{\text {soil }}=0 \cdot 3, \eta_{\text {soil }}=5 \%$ and $k_{h}=0 \cdot 1$.

the displacement, velocity and acceleration-time history response for a particular case with $t_{1}=5 \mathrm{~s}$, wall height, $H=5 \mathrm{~m}$, concrete unit weight for the wall, $\gamma_{\text {conc }}=24 \mathrm{kN} / \mathrm{m}^{3}$, shear modulus of concrete wall, $G_{\text {conc }}=9921 \times 10^{3} \mathrm{kN} / \mathrm{m}^{2}$, Poisson's ratio of concrete wall, $\mu_{\text {conc }}=0 \cdot 26$, damping factor for concrete wall, $\eta_{\text {conc }}=5 \%$, backfill sand with unit weight, $\gamma_{\text {soil }}=18 \mathrm{kN} / \mathrm{m}^{3}$, shear modulus of backfill, $G_{\text {soil }}=5769 \mathrm{kN} / \mathrm{m}^{2}$, Poisson's ratio of backfill, $\mu_{\text {soil }}=0 \cdot 3$, damping factor for soil, $\eta_{\text {soil }}=20 \%$ and horizontal seismic acceleration coefficient, $k_{h}=0.32$ is obtained.

Again, the displacement, velocity and acceleration-time history response for the same wall and backfill material with $t_{1}=5 \mathrm{~s}$, damping factor for soil, $\eta_{\text {soil }}=5 \%$ and $k_{h}=0.1$ is shown in figure 6.

Now the maximum active earth pressure force, $\left|Q_{b}\right|$ on the retaining wall is computed for the harmonically excited system using (4). The total active earth force value is normalized with respect to the term $\left(\rho \ddot{x}_{g} H^{2}\right)$ and plotted with various values of the frequency ratio, $\omega / \omega_{1}$, where $\omega$ is the circular frequency of excitation and $\omega_{1}$ is the fundamental circular natural frequency of the stratum when it is considered to respond as a cantilever shear-beam. For a homogeneous medium it is given by,

$$
\omega=\pi v_{s} / 2 H,
$$

where $v_{s}$ is the shear wave velocity for the medium and is given by $G / \rho^{1 / 2}$.

Finally, the non-dimensional results are plotted in figure 7 for three different values of damping factors for soil $\left(\eta_{\text {soil }}\right)$ viz. 5, 10 and $20 \%$ respectively. 


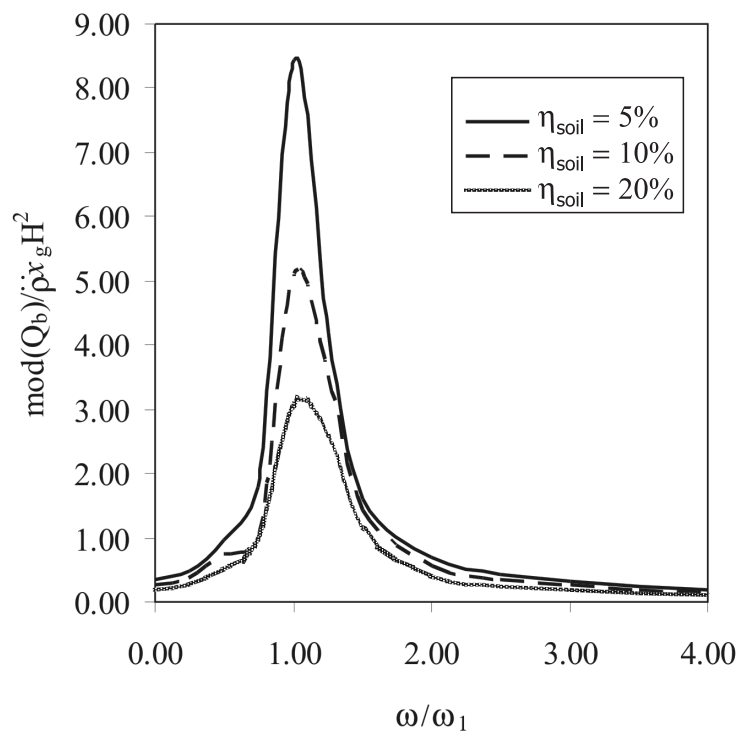

Figure 7. Effect of damping on nondimensional dynamic earth force with harmonically excited system for $\mu_{\text {soil }}=$ $0 \cdot 3$

\section{Discussions and comparison of results}

From the displacement-time history curve as shown in figure 5, it can be seen that the maximum displacement ranges around $0.2 \mathrm{~mm}$, which is about $4 \%$ of the total height of the wall, for a wall height, $H=5 \mathrm{~m}$, unit weight of concrete wall, $\gamma_{\text {conc }}=24 \mathrm{kN} / \mathrm{m}^{3}$, shear modulus of concrete wall, $G_{\text {conc }}=9921 \times 10^{3} \mathrm{kN} / \mathrm{m}^{2}$, Poisson's ratio of concrete wall, $\mu_{\text {conc }}=0 \cdot 26$, damping factor for concrete wall, $\eta_{\text {conc }}=5 \%$, backfill sand with unit weight, $\gamma_{\text {soil }}=18 \mathrm{kN} / \mathrm{m}^{3}$, shear modulus of backfill, $G_{\text {soil }}=5769 \mathrm{kN} / \mathrm{m}^{2}$, Poisson's ratio of backfill, $\mu_{\text {soil }}=0 \cdot 3$, damping factor for soil, $\eta_{\text {soil }}=20 \%$ and horizontal seismic acceleration coefficient, $k_{h}=0.32$. While the same for the similar wall and backfill with damping factor for soil, $\eta_{\text {soil }}=5 \%$ and $k_{h}=0 \cdot 1$ is around $0.1 \mathrm{~mm}$ (see figure 6), which is about $2 \%$ of the total height of the wall. Similar to the observation of Scott (1973), here also these results compare well with the assumption of the present model, which is valid for small displacements only.

Variation of the normalized earth force with various values of damping factors is shown in figure 7. It can be seen that for each case the normalized value of the earth force attains its maximum value at $\omega / \omega_{1}=1$, as expected. The maximum value of the normalized earth force decreases with increase in damping factors, as shown in figure 7. For example, when the damping factor increases from 5 to $10 \%$ the maximum value of the normalized earth force decreases by about $39.5 \%$. Again, when the damping factor increases from 10 to $20 \%$ the maximum value of the normalized earth force decreases by about $40 \%$. However, at other frequency ratios, the variation in the value of normalized earth force is relatively small with different damping factors, as expected. This proposed design chart as shown in figure 7 can easily be used to obtain the total earth force on the wall under seismic condition.

To validate the proposed 2-DOF model, the present results are compared with the results of Scott's (1973) model with Poisson's ratio, $\mu_{\text {soil }}=0 \cdot 3$ and damping factor for soil, $\eta_{\text {soil }}=10 \%$. The comparison is shown in figure 8. From the figure it can be seen that the present result compares well with that of Scott's (1973) model. As already mentioned by other researchers like Wood (1973), Veletsos \& Younan (1994), one of the major shortcoming of Scott's (1973) 


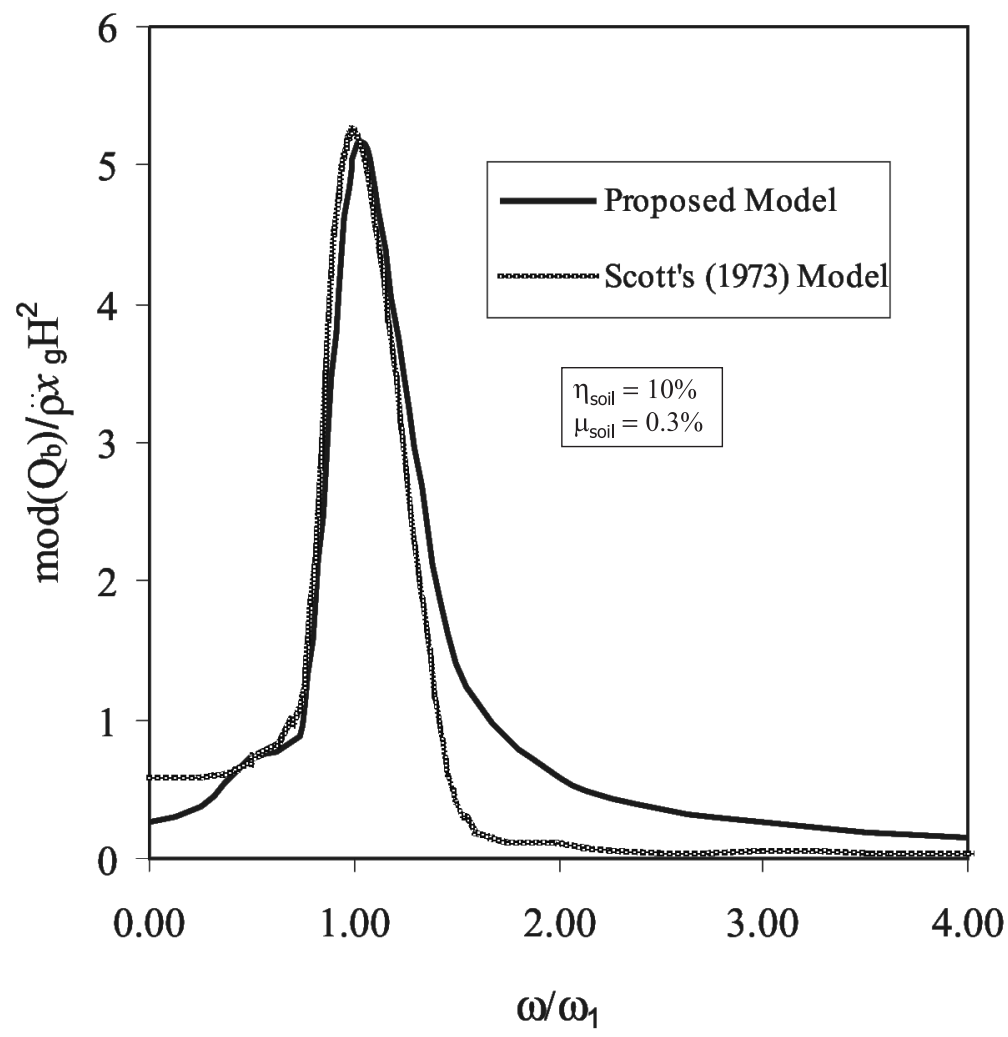

Figure 8. Comparison of present result with Scott's (1973) model.

model is that it underestimates the earth force on the wall at higher values of frequency ratio, which has been corrected by the present analysis using a better 2-DOF model instead of the conventional SDOF model. The difference in the results of the present study and those of Scott (1973) can be attributed to the selection of a better and more realistic mathematical model in the present study.

\section{Conclusions}

The main conclusions of the present analysis can be summarized as follows

(1) Total dynamic earth pressure force on the vertical retaining wall, which retains a semiinfinite, homogeneous, viscoelastic medium of soil, can easily be calculated using the proposed 2-DOF mass-spring-dashpot model compared to the conventional SDOF model. Also the critical distance of influence zone for the dynamic earth pressure is obtained by the present study.

(2) The displacement, velocity and acceleration-time history for any input earthquake motion can easily be obtained which in turn finally gives the amount of wall movement required for the design. 
(3) The non-dimensional design chart proposed from the present study can easily be used to compute the total dynamic earth force acting on the retaining wall for different input ground motion and damping properties of the backfill.

(4) Present results compare well with the existing Scott's (1973) model, however, the major shortcoming of the conventional Scott (1973) model to estimate the earth force at higher frequency is corrected by the proposed method.

\section{List of symbols}

$\begin{array}{ll}c & \text { damping constant; } \\ c_{1}, c_{2} & \text { damping constant for soil and wall respectively; } \\ g & \text { acceleration due to gravity; } \\ G & \text { shear modulus; } \\ G_{\text {soil }}, G_{\text {conc }} & \text { shear modulus of soil and concrete respectively; } \\ H & \text { height of the wall; } \\ k & \text { stiffness; } \\ k_{1}, k_{2} & \text { stiffness of soil and wall respectively; } \\ k_{h} & \text { horizontal seismic acceleration coefficient; } \\ m & \text { mass; } \\ M_{1}, M_{2} & \text { mass of soil and wall respectively; } \\ \text { mod } Q_{b}=\left|Q_{b}\right| & \text { total earth force on the wall; } \\ t_{1} & \text { finite time; } \\ \ddot{x}_{g} & \text { amplitude of ground acceleration; } \\ \gamma_{\text {soil }}, \gamma_{\text {conc }} & \text { unit weight of soil and concrete respectively; } \\ \eta & \text { damping factor; } \\ \eta_{\text {soil }}, \eta_{\text {conc }} & \text { damping factor for soil and concrete wall; } \\ \mu_{\text {soil }}, \mu_{\text {conc }} & \text { Poisson's ratio of soil and concrete; } \\ v_{s} & \text { shear wave velocity; } \\ \rho & \text { mass density; } \\ \omega & \text { circular frequency of excitation; } \\ \omega_{1} & \text { fundamental circular natural frequency. }\end{array}$

\section{References}

Chopra A K 2003 Dynamics of structure theory and application to earthquake engineering 2nd edn (New Delhi: Prentice Hall of India)

Choudhury D, Singh S 2006 New approach for estimation of static and seismic active earth pressure. Geotech. Geol. Eng. 24: 117-127

Choudhury D, Subba Rao K S 2002 Displacement related active earth pressure. Proc. Int. Conf. on Advances in Civil Engineering (ACE 2002) 2: 1038-1047

Kramer S L 1996 Geotechnical earthquake engineering (NJ: Englewood Cliffs, Prentice Hall)

Mononobe N, Matsuo H 1929 On the determination of earth pressures during earthquakes. Proc. World Engineering Conference 9: 176

Okabe S 1926 General theory of earth pressure. J. Jap. Soc. Civil Eng. (Tokyo) 12(1):

Saran S, Gupta R P 2003 Seismic earth pressures behind retaining walls. Indian Geotech. J. 33: 195213 
Scott R F 1973 Earthquake-induced pressures on retaining walls. Proc. 5th World Conf. on Earthquake Engineering (Tokyo: Int. Assoc. Earthquake Eng.) 2: 1611-1620

Subba Rao K S, Nayak S, Choudhury D 2004 Determination of displacement-related passive earth pressure. Geotech. Eng. J. 35(2): 79-85

Veletsos A S, Younan A H 1994a Dynamic modeling and response of soil-wall systems. J. Geotech. Eng. ASCE 120: 2155-2179

Veletsos A S, Younan A H 1994b Dynamic soil pressures on rigid vertical walls. Earthquake Eng. Struct. Dyn. 23: 275-301

Wood J H 1973 Earthquake-induced pressures on retaining walls. Rep. EERL 73-05, Earthquake Eng. Res. Lab., California Institute of Technology, Pasadena, CA 